\title{
Space-Time-Frequency Diversity for OFDM-Based Indoor Power Line Communication
}

\author{
Gokce HACIOGLU \\ Dept. of Electrical and Electronics Engineering, Karadeniz Technical University, Universite M., 61080 Trabzon \\ gokcehacioglu@ktu.edu.tr
}

\begin{abstract}
In this paper, full rate space-time-frequency coding applied to orthogonal frequency division multiplexing based power line communication systems. The proposed systems yield both time and frequency diversity and keep transmission rate full. Performances of the systems are evaluated for three conductors of low voltage indoor cables and are compared with space-frequency and space-time-frequency coding applied power line communication systems in the literature. Owing to the higher order diversity level the proposed full rate space-time-frequency systems have an increasing advantage over space-frequency systems as the SNR level above $12.5 \mathrm{~dB}$. On the other hand, owing to transmission rate advantage, the proposed full rate space-time-frequency systems can have more than $6 \mathrm{~dB}$ gain over the other space-time-frequency coding applied power line communication systems in the literature.
\end{abstract}

\section{Keywords}

Power line communication, constellation rotation, space-time-frequency (STF) diversity

\section{Introduction}

Power lines offer broadband communication in an affordable way; however, power distribution systems are not designed to carry high frequencies. Unmatched loads and joint points cause a multipath propagation and a frequency selective fading [1]. Power line communication (PLC) channels cause a strong inter symbol interference (ISI), and therefore an expensive equalizer may be needed for a PLC modem. Fortunately, an orthogonal frequency division multiplexing (OFDM) can be used to convert a frequency selective channel to many parallel flat fading channels [2]. Therefore, a single tap cheap equalizer can be enough for a PLC modem through the use of OFDM. The noise in PLC systems has different properties in comparison with conventional communication systems. Especially an impulsive noise in the PLC can take very large amplitude values. A frequency selective fading and the noise can degrade performances of PLC systems significantly [3].
Diversity methods have been used to overcome negative effects of fading in wireless communications. The diversity methods need uncorrelated or at least low correlated channels [4]. The PLC systems have the capability of using multiple lines to obtain uncorrelated channels [5]. The channel behaviors of these multiple lines can be almost the same [6]. Therefore, space diversity methods are not sufficient for power lines. However frequency and time domain can be used to get a diversity gain for the PLC [7], [8].

Space-time block coding, which is a space diversity method, has been used to employ transmitting diversity for wireless systems [9]. Space-time block coding can be used in a different manner to achieve the time and frequency diversity for PLC systems [8]. On the other hand, the PLC systems are also suitable for using both transmitting and receiving diversity methods. Maximal ratio combining (MRC) can be applied easily to provide diversity gain in PLC systems [7].

Detection complexity, data transmission rate and the cost are the key factors that should be taken into account during a diversity method design. If a diversity method needs detecting symbols jointly, it causes increase of detection complexity. A space-time block coding can provide only second order transmit diversity without causing data rate loss or increasing detection complexity [10]. Second order space-time block code named as Alamouti code is the first space-time block code [11]. Constellation rotation can employ second order of transmit diversity without an increase in a detection complexity [12] and also provide time, frequency or space diversity [13]. If constellation rotation and space-time block coding are used together, a fourth order of diversity can be employed without increase in detection complexity [12], [13].

The proposed systems are aimed to be used for a single phase low voltage cable consisting of three wires. Two independent differential signaling can be used via feeding three of the possibilities: Phase-neutral, phase-protective earth and neutral-protective earth. In this study, the diversity method, which is proposed in [13] applied to OFDM based power line communication. Both receive and transmit diversity applications of diversity method in [13] are showed for OFDM based PLC. The proposed systems are 
compared with space-time block coding applied and maximal ratio combining applied OFDM based power line communication systems in the literature. The proposed systems are able to obtain second order frequency and second order time diversity without increasing detection complexity or causing data rate loss. On the other hand the systems proposed in [8] and [14] cause data rate loss. Therefore, the proposed systems are referred to as rate 1 and the systems in [8] and [14] as rate $1 / 2$. According to simulation results, the proposed systems have more diversity order than space-frequency methods and more gain than space-time-frequency diversity methods in the literature.

This paper is organized as follows: In Sec. 2, the power line channel and impulsive noise models are described. In Sec. 3, the system model and applications of full rate space-time-frequency methods for power line communications are presented. In Sec. 4, simulation results and in Sec. 5, some conclusions are given.

Notation: Bold characters are used for vectors and matrices, ${ }^{*}$ denotes the complex conjugate, $|\mathbf{X}|$ denotes magnitude or Euclidean norm of vector $\mathbf{X}, \operatorname{diag}(\mathbf{X})$ denotes a diagonal matrix where the diagonal entries are elements of $\mathbf{X}$, PLC denotes power line communication, STF denotes space-time-frequency, SF denotes space-frequency, MRC denotes maximal ratio combining and AWCN denotes additive white class A noise.

\section{The Power Line Channel and Noise Model}

There are two approaches to model a power line channel which are namely the top-down and the bottom-up approaches. The bottom-up approach models the channel behavior via characteristics of components in the network [15], [16]. However it has a computational complexity. The top-down approach has the parameters that are obtained by the measurements and this approach has less computation complexity. In this study, a PLC channel model, obtained by a top-down approach, is used. A deterministic PLC channel frequency response was obtained by the top-down approach in [1] and it is given below:

$$
H(f)=\sum_{i=1}^{N_{p}} g_{i} \exp \left(-\left(a_{0}+a_{1} f^{k}\right) d_{i}\right) \exp \left(-\frac{j 2 \pi f d_{i}}{v_{p}}\right)
$$

where $N_{p}, g_{i}, a_{0}$, and $a_{1}, d_{i}, v_{p}$ denote number of paths, weighting factor of path $i$, attenuation terms, length of path $i$ and the speed of electromagnetic wave in the wire, respectively. On the other hand, a PLC channel can change with time due to time variant loads or topology changes. Therefore, the channel model should include randomness. A top-down random channel generator proposed in [17] is used for the simulations. The random channel model can be represented as given below:

$$
H(f)=B \sum_{i=1}^{N_{p}}\left(g_{i}+c_{i} f^{K_{2}}\right) \exp \left(-\left(a_{0}+a_{1} f^{K}\right) d_{i}\right) \exp \left(-\frac{j 2 \pi f d_{i}}{v_{p}}\right)
$$

where $B, a_{0}, a_{1}, K, K_{2}, v_{p}$ are fixed and $g_{i}, c_{i}, d_{i}, N_{p}$ are the random parameters. The $g_{i}$ and $c_{i}$ are zero mean log-normal distributed random variables with variances $\sigma_{g}^{2}$ and $\sigma_{c}^{2}=b_{0} \sigma_{g}^{2}$ respectively. The path lengths $d_{i}$ are uniformly distributed over $[0, L]$ where $L$ is constant. The number of paths $N_{p}$ is a Poisson random variable with mean $\Lambda L$.

The broadband characteristics of PLC channel was measured up to $100 \mathrm{MHz}$ and reported in [18]. According to the measurement results in [18], 9 classes of PLC channels were defined and the class 3 channel was reported as more probable to occur. In this paper, class 3 channel parameters are used in simulations. Table 1 shows parameters to use channel model for class 3 channels [17].

\begin{tabular}{|c|c|c|c|c|}
\hline$B$ & $a_{0}\left(\mathrm{~m}^{-1}\right)$ & $a_{1}\left(\mathrm{~s} \mathrm{~m}^{-1}\right)$ & $K$ & $L(\mathrm{~m})$ \\
\hline $6.7170 \times 10^{-4}$ & -0.0152108 & $3.67885 \cdot 10^{-5}$ & 0.347786 & 320 \\
\hline
\end{tabular}

Tab. 1. Channel parameters for class-3 channel.

On the other hand, $b_{0}^{2}=0, \sigma_{g}^{2}=1, \Lambda=0.2$ paths $/ \mathrm{m}, v_{p}=$ $2 \cdot 10^{8} \mathrm{~m} / \mathrm{s}$ and $K_{2}=0$ are the values that were used as fixed parameters in the channel model [17].

Power lines have five different noise sources [3] which are denoted as: colored background noise, narrowband noise, periodic impulsive noise asynchronous to mains frequency, periodic impulsive noise synchronous to mains frequency and asynchronous impulsive noise. In this study, it is assumed that noise is impulsive and a Middleton Class A noise model is used in the simulations [19]. The probability density function (PDF) of Middleton Class A noise is given below.

$$
\begin{aligned}
f_{X}(x) & =\exp (-A) \sum_{m=0}^{\infty} \frac{A^{m}}{m ! \sqrt{2 \pi \sigma_{m}^{2}}} \exp \left(-\frac{x^{2}}{2 \sigma_{m}^{2}}\right) \\
& =\sum_{m=0}^{\infty} f_{P}(p) f_{G}(g) .
\end{aligned}
$$

The parameter $A$ is named as impulsive index. If $A$ becomes larger, the PDF of noise converges to Gaussian. On the other hand, if $A$ becomes smaller, noise characteristics converge to impulsive noise. The $\sigma_{m}^{2}$ can be obtained as follows:

$$
\sigma_{m}^{2}=\frac{\sigma^{2}\left(\frac{m}{A}+\Gamma\right)}{1+\Gamma}=\frac{m \sigma_{I}^{2}}{A}+\sigma_{G}^{2}
$$

where $\sigma_{G}^{2}, \sigma_{I}^{2}, \sigma^{2}=\sigma_{G}^{2}+\sigma_{I}^{2}$ and $\Gamma=\frac{\sigma_{G}^{2}}{\sigma_{I}^{2}}$ denote variance of Gaussian noise, variance of the impulsive noise, variance of noise, the ratio between power of Gaussian noise and impulsive noise power, respectively. The probability density functions, $f_{P}(p)$ and $f_{G}(g)$ are Poisson and 
Gaussian density functions, respectively. The mean of Poisson distributed random variable $p$ is $A$. The mean and the variance values of Gaussian distributed random variable $g$ are 0 and $\sigma_{G}{ }^{2}$, respectively. The parameter $A$ is also equal to $V_{t} T_{s}$ where $V_{t}$ is mean impulse rate and $T_{s}$ is impulse duration.

\section{The System Model}

A broadband PLC system is considered so that the PLC system uses OFDM to reduce inter-symbol interference. After removing cyclic prefix and applying Discrete Fourier Transform, the receive vector of the system is stated as below:

$$
\begin{array}{r}
\mathbf{Y}=\underbrace{\left[\begin{array}{ccccc}
H^{t}[0] & 0 & \ldots & \ldots & 0 \\
0 & H^{t}[1] & 0 & \ldots & 0 \\
\vdots & 0 & \ddots & \ldots & \vdots \\
\vdots & \vdots & \ddots & \ddots & 0 \\
0 & 0 & \ldots & 0 & H^{t}[N-1]
\end{array}\right]}_{\mathbf{H}^{t}}\left[\begin{array}{c}
S[0] \\
S[1] \\
\vdots \\
\vdots \\
S[N-1]
\end{array}\right]+\mathbf{N} \\
\mathbf{H}^{\mathbf{t}}=\operatorname{diag}\left(\left[\begin{array}{c}
H^{t}[0] \\
H^{t}[1] \\
\vdots \\
H^{t}[N-1]
\end{array}\right]\right)
\end{array}
$$

where $S[k], H^{t}[k]$ denote transmitted symbols and the channel coefficients, respectively, $\mathbf{H}^{t}$ denotes channel matrix and $t$ denotes time instant. If the carrier frequency is $f_{c}$ and subcarrier spacing is $\Delta f$; the channel coefficients can be written as follows:

$$
H^{t}[k]=\left.H^{t}(f)\right|_{f=f_{c}+k \Delta f} ; k=0,1, \ldots, N-1
$$

$H^{t}(f)$ can be obtained by using (2). The noise vector is denoted by $\mathbf{N}$ and can be written as follows:

$$
\mathbf{N}=\frac{1}{\sqrt{N}}\left[\begin{array}{ccccc}
1 & 1 & 1 & \ldots & 1 \\
1 & W_{N} & W_{N}^{2} & \ldots & W_{N}^{N-1} \\
\vdots & \vdots & \vdots & \vdots & \vdots \\
1 & W_{N}^{N-1} & W_{N}^{2(N-1)} & \ldots & W_{N}^{(N-1)^{2}}
\end{array}\right] \underbrace{\left[\begin{array}{c}
\tilde{n}[0] \\
\tilde{n}[1] \\
\vdots \\
\tilde{n}[N-1]
\end{array}\right]}_{\widetilde{\mathbf{N}}}=\underbrace{\left[\begin{array}{c}
n[0] \\
n[1] \\
\vdots \\
n[N-1]
\end{array}\right]}_{\mathbf{N}}
$$

where $W_{N}=\exp (-j 2 \pi / N)$ and $\tilde{n}[0], \tilde{n}[1], \ldots, \tilde{n}[N-1]$ are Middleton Class A noise terms. The elements of noise vector $\mathbf{N}$ are also Middleton Class A distributed because the autocorrelations of $\mathbf{N}$ and $\tilde{\mathbf{N}}$ are the same.

It is assumed that two transmit channels are employed by using multiple conductors in the power line and therefore a low voltage power cable consisting of 1 phase, 1 neutral and 1 ground wires is assumed. Two of the wires can be used to transfer data simultaneously and the other can perform as a return path. The channel coefficient be- tween transmit channel $i$ and the receive point $j$ is denoted as $H_{i j}^{t}(f)$. It is assumed that a strong correlation exists between wires. Therefore $H_{11}^{t}(f)=H_{22}^{t}(f)=H^{t}(k)$ is applied in the simulations. However crosstalk is not considered. The average attenuation model for class- 3 channel is given in [18] and formulated as below

$$
|H(f)|_{\text {average }}(\mathrm{dB})=-38+25 \exp \left(-\frac{f}{3 \cdot 10^{6}}\right)-\frac{14}{10^{8}} f .
$$

According to the average channel attenuation and the simulation model toward high frequencies, channel attenuates more as shown in Fig. 1. Therefore, symbols are flipped to transmit them both by higher and by lower frequencies to obtain the frequency diversity gain. The channel matrices for the proposed systems can be written as follows:

$$
\left.\left.\begin{array}{rl}
\mathbf{H}_{1}^{t}=\operatorname{diag}\left(\left[\begin{array}{c}
H^{t}[0] \\
H^{t}[1] \\
\vdots \\
H^{t}[N-1]
\end{array}\right]\right), \\
\mathbf{H}_{2}^{t}=\operatorname{diag}\left(\left[\begin{array}{c}
H^{t}[N-1] \\
H^{t}[N-2] \\
\vdots \\
H^{t}[0]
\end{array}\right]\right), \\
\mathbf{H}_{3}^{t+m T}=\operatorname{diag}\left(\left[\begin{array}{c}
H^{t+m T}\left[\frac{N}{2}-1\right] \\
H^{t+m T}\left[\frac{N}{2}-2\right] \\
H^{t+m T}[0] \\
H^{t+m T}[N-1] \\
H^{t+m T}[N-2] \\
\vdots \\
H^{t+m}
\end{array}\right]\right) \\
H^{t+m T}\left[\frac{N}{2}\right] \\
H^{t+m T}\left[\frac{N}{2}\right] \\
H^{t+m T}\left[\frac{N}{2}+1\right.
\end{array}\right]\right),
$$

where $T$ denotes symbol period. 


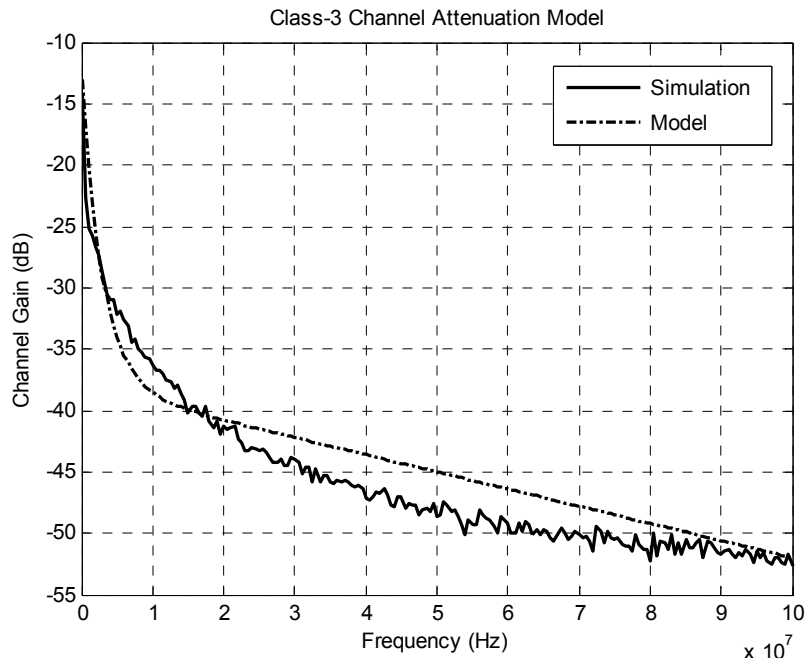

Fig. 1. Average attenuation model for class-3 channel.

It is assumed that the channel is changed in $m T$ seconds or $m T$ is bigger than the coherence time of the channel which provides acquiring time diversity gain. Each of the symbols is transmitted by four different subcarriers in two distinct time slots to obtain time and frequency diversity. Rotated QPSK constellation is applied in the simulations. The major advantage of a rotated constellation is the identifiability of the symbols by their real or imaginary parts. The rotated constellation is obtained by multiplying each of the constellation points by $\exp (j \phi)$. The phase angle $\phi=30^{\circ}$ value is used in the simulations.

$S_{i R, k}, S_{i I, k}$ denote $k^{\text {th }}$ transmit symbols, where $i=1,2$. The transmit symbols obtained as follows:

$$
\begin{gathered}
S_{i R, k}=\operatorname{Re}\left\{S_{2 i-1}[k]\right\}+j \cdot \operatorname{Re}\left\{S_{2 i}[k]\right\}, \\
S_{i l, k}=\operatorname{Im}\left\{S_{2 i-1}[k]\right\}+j \cdot \operatorname{Im}\left\{S_{2 i}[k]\right\} ; i=1,2
\end{gathered}
$$

where $S_{1}[k], S_{2}[k], S_{3}[k]$ and $S_{4}[k]$ denote four independent symbols which are elements of rotated QPSK constellation. The transmit symbols are transmitted through four channels.

In this study, two systems are proposed to obtain space-time-frequency diversity while keeping the transmission rate full. The proposed systems are named as proposed system-I (PS-I) and proposed system-II (PS-II). The proposed systems are compared with SF and STF diversity applied power line communication systems which are given in [5] and [8], respectively. As Alamouti coding is in use, the receive vectors of the SF diversity system is indicated as given below.

$$
\mathbf{Y}_{1 A S F}=\mathbf{H}_{1}^{t}\left[\begin{array}{c}
S_{1}[0] \\
S_{1}[1] \\
\vdots \\
S_{1}[N-1]
\end{array}\right]+\mathbf{H}_{2}^{t}\left[\begin{array}{c}
S_{2}[0] \\
S_{2}[1] \\
\vdots \\
S_{2}[N-1]
\end{array}\right]+\mathbf{N}_{1}
$$

$$
\mathbf{Y}_{2 A S F}=-\mathbf{H}_{1}^{t+T}\left[\begin{array}{c}
S_{2}^{*}[0] \\
S_{2}^{*}[1] \\
\vdots \\
S_{2}^{*}[N-1]
\end{array}\right]+\mathbf{H}_{2}^{t+T}\left[\begin{array}{c}
S_{1}^{*}[0] \\
S_{1}^{*}[1] \\
\vdots \\
S_{1}^{*}[N-1]
\end{array}\right]+\mathbf{N}_{2} .
$$

The receive vectors of the SF diversity during the MRC used are given below.

$$
\begin{gathered}
\mathbf{Y}_{1 M S F}=\mathbf{H}_{1}^{t}\left[\begin{array}{c}
S_{1}[0] \\
S_{1}[1] \\
\vdots \\
S_{1}[N-1]
\end{array}\right]+\mathbf{N}_{1}, \\
\mathbf{Y}_{2 M S F}=\mathbf{H}_{2}^{t}\left[\begin{array}{c}
S_{1}[0] \\
S_{1}[1] \\
\vdots \\
S_{1}[N-1]
\end{array}\right]+\mathbf{N}_{2} .
\end{gathered}
$$

The proposed systems are also compared with rate $1 / 2$ STF diversity systems. The receive vectors of rate $1 / 2$ STF diversity system with Alamouti coding used is indicated as given below.

$\mathbf{Y}_{1 A S T F}=\mathbf{H}_{1}^{t}\left[\begin{array}{c}S_{1}[0] \\ S_{1}[1] \\ \vdots \\ S_{1}[N-1]\end{array}\right]+\mathbf{H}_{2}^{t}\left[\begin{array}{c}S_{2}[0] \\ S_{2}[1] \\ \vdots \\ S_{2}[N-1]\end{array}\right]+\mathbf{N}_{1}$,

$\mathbf{Y}_{2 A S T F}=-\mathbf{H}_{1}^{t+T}\left[\begin{array}{c}S_{2}^{*}[0] \\ S_{2}^{*}[1] \\ \vdots \\ S_{2}^{*}[N-1]\end{array}\right]+\mathbf{H}_{2}^{t+T}\left[\begin{array}{c}S_{1}^{*}[0] \\ S_{1}^{*}[1] \\ \vdots \\ S_{1}^{*}[N-1]\end{array}\right]+\mathbf{N}_{2}$

$\mathbf{Y}_{3 A S T F}=\mathbf{H}_{3}^{t+m T}\left[\begin{array}{c}S_{1}[0] \\ S_{1}[1] \\ \vdots \\ S_{1}[N-1]\end{array}\right]+\mathbf{H}_{4}^{t+m T}\left[\begin{array}{c}S_{2}[0] \\ S_{2}[1] \\ \vdots \\ S_{2}[N-1]\end{array}\right]+\mathbf{N}_{3}$,

$$
\mathbf{Y}_{4 A S T F}=-\mathbf{H}_{3}^{t+(m+1) T}\left[\begin{array}{c}
S_{2}^{*}[0] \\
S_{2}^{*}[1] \\
\vdots \\
S_{2}^{*}[N-1]
\end{array}\right]+-\mathbf{H}_{4}^{t+(m+1) T}\left[\begin{array}{c}
S_{1}^{*}[0] \\
S_{1}^{*}[1] \\
\vdots \\
S_{1}^{*}[N-1]
\end{array}\right]+\mathbf{N}_{4} .
$$

The receive vectors of rate $1 / 2$ STF diversity system with the MRC used are indicated as follows.

$$
\mathbf{Y}_{1 M S T F}=\mathbf{H}_{1}^{t}\left[\begin{array}{c}
S_{1}[0] \\
S_{1}[1] \\
\vdots \\
S_{1}[N-1]
\end{array}\right]+\mathbf{N}_{1},
$$




$$
\begin{gathered}
\mathbf{Y}_{2 M S T F}=\mathbf{H}_{2}^{t}\left[\begin{array}{c}
S_{1}[0] \\
S_{1}[1] \\
\vdots \\
S_{1}[N-1]
\end{array}\right]+\mathbf{N}_{2}, \\
\mathbf{Y}_{3 M S T F}=\mathbf{H}_{3}^{t+m T}\left[\begin{array}{c}
S_{1}[0] \\
S_{1}[1] \\
\vdots \\
S_{1}[N-1]
\end{array}\right]+\mathbf{N}_{3}, \\
\mathbf{Y}_{4 M S T F}=\mathbf{H}_{4}^{t+m T}\left[\begin{array}{c}
S_{1}[0] \\
S_{1}[1] \\
\vdots \\
S_{1}[N-1]
\end{array}\right]+\mathbf{N}_{4} .
\end{gathered}
$$

\subsection{The Proposed System-I}

Alamouti coding and constellation rotation are used together for the proposed system-I (PS-I). The receive vectors of the proposed system-I are given below,

$$
\begin{gathered}
\mathbf{Y}_{1}=\mathbf{H}_{1}^{t}\left[\begin{array}{c}
S_{1 R, 0} \\
S_{1 R, 1} \\
\vdots \\
S_{1 R, N-1}
\end{array}\right]+\mathbf{H}_{2}^{t}\left[\begin{array}{c}
S_{2 R, 0} \\
S_{2 R, 1} \\
\vdots \\
S_{2 R, N-1}
\end{array}\right]+\mathbf{N}_{1}, \\
\mathbf{Y}_{2}=-\mathbf{H}_{1}^{t+T}\left[\begin{array}{c}
S_{2 R, 0}^{*} \\
S_{2 R, 1}^{*} \\
\vdots \\
S_{2 R, N-1}^{*}
\end{array}\right]+\mathbf{H}_{2}^{t+T}\left[\begin{array}{c}
S_{1 R, 0}^{*} \\
S_{1 R, 1}^{*} \\
\vdots \\
S_{1 R, N-1}^{*}
\end{array}\right]+\mathbf{N}_{2}, \\
\mathbf{Y}_{3}=\mathbf{H}_{3}^{t+m T}\left[\begin{array}{c}
S_{1 I, 0} \\
S_{1 I, 1} \\
\vdots \\
S_{1 I, N-1}
\end{array}\right]+\mathbf{H}_{4}^{t+m T}\left[\begin{array}{c}
S_{2 I, 0} \\
S_{2 I, 1} \\
\vdots \\
S_{2 I, N-1}
\end{array}\right]+\mathbf{N}_{3}, \\
\mathbf{Y}_{4}^{*}=-\mathbf{H}_{3}^{t+(m+1) T}\left[\begin{array}{c}
S_{1 I, 0}^{*} \\
S_{2 I, 0}^{*} \\
S_{2 I, 1}^{*} \\
\vdots \\
S_{2 I, N-1}^{*}
\end{array}\right]+\mathbf{H}_{4}^{t+(m+1) T}\left[\begin{array}{c}
S_{1 I, 1} \\
\vdots \\
S_{1 I, N-1}^{*}
\end{array}\right]+\mathbf{N}_{4}
\end{gathered}
$$

where real parts of the four symbols coded by Alamouti coding and transmitted in two symbol periods and the receive vectors $\mathbf{Y}_{1}$ and $\mathbf{Y}_{2}$ are obtained in return at the receiver. Similarly the receive vectors $\mathbf{Y}_{3}$ and $\mathbf{Y}_{4}$ are obtained for imaginary parts of the four symbols in two symbol periods. The channel is assumed as staying constant at least during two symbol periods. Hence the channel matrices $\mathbf{H}^{t}$ and $\mathbf{H}^{t+T}$ are assumed as equal to each other. Two transmit channels are generated by using (2) in the simulations. Four transmit channels are obtained by using (10), (11),
(12) and (13). Consequently, a second order of frequency and a second order of time diversity are yielded. The detection of the symbol $S_{1}[0]$ is made as following:

$$
\begin{gathered}
\widetilde{S}_{1}[0]=\min _{S_{n}}\left(\left|\mathbf{Y}_{1}-\mathbf{H}_{1}^{t}\left[\begin{array}{c}
\operatorname{Re}\left\{S_{n}\right\} \\
0 \\
\vdots \\
0
\end{array}\right]\right|^{2}+\mathbf{Y}_{2}-\left.\mathbf{H}_{2}^{t+T}\left[\begin{array}{c}
\operatorname{Re}\left\{S_{n}\right\} \\
0 \\
\vdots \\
0
\end{array}\right]\right|^{2}+\right. \\
\mathbf{Y}_{3}-\left.\mathbf{H}_{3}^{t+m T}\left[\begin{array}{c}
j \cdot \operatorname{Im}\left\{S_{n}\right\} \\
0 \\
\vdots \\
0
\end{array}\right]\right|^{2}\left|\mathbf{Y}_{4}-\mathbf{H}_{4}^{t+(m+1) T}\left[\begin{array}{c}
-j \cdot \operatorname{Im}\left\{S_{n}\right\} \\
0 \\
\vdots \\
0
\end{array}\right]\right|^{2}|| \mid
\end{gathered}
$$

where $S_{n} ; n=0,1,2,3$ are the elements of rotated QPSK constellation. The other symbols can be detected in a similar way.

Figure 2 shows a general block diagram of spacefrequency or space-time-frequency PLC systems as Alamouti coding in use.

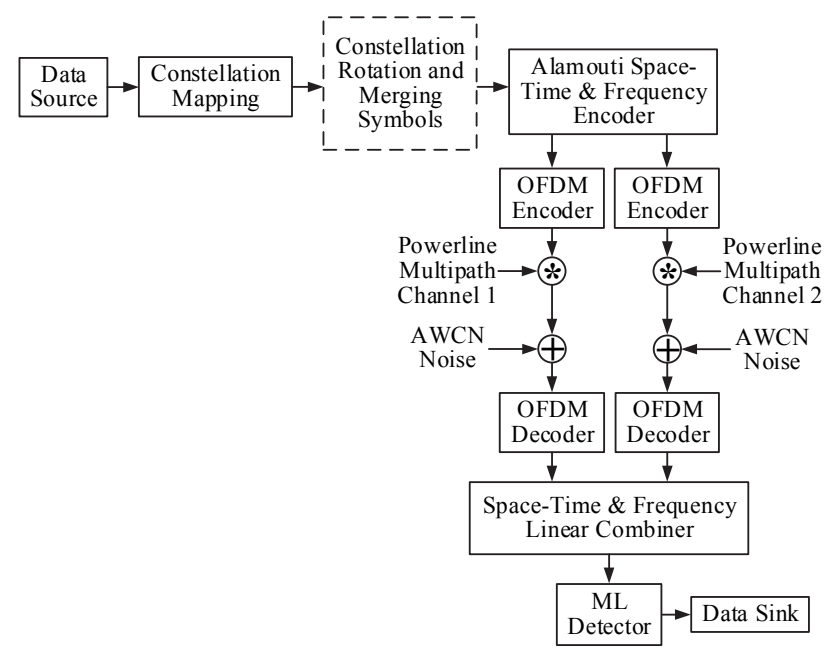

Fig. 2. Block diagram of space-frequency or space-timefrequency diversity systems as Alamouti coding in use.

AWCN denotes Middleton's additive white class A noise and ML detector denotes maximum likelihood detector. The proposed system-I includes all of the blocks in Fig. 2. However conventional systems do not include the dashed line block in Fig. 2. Alamouti Space-Time \& Frequency Encoder in Fig. 2 encodes symbols to obtain; rate-1 spacefrequency, rate $1 / 2$ space-time-frequency or the proposed rate-1 space-time-frequency diversity.

\subsection{The Proposed System-II}

Space-time block coding was proposed to reduce size and power consumption of mobile receiver in wireless communication. Besides, power-line communication receiver has more flexibility on the size and power consumption. Therefore MRC can be used instead of Alamouti coding in PLC systems. MRC has $3 \mathrm{~dB}$ more gain than 
Alamouti coding in wireless systems [11]. However the need of two transmitters for both of the Alamouti coding and MRC in PLC systems cancels the $3 \mathrm{~dB}$ gain advantage of MRC. Rotated constellation and maximal ratio combining are applied in the proposed system-II.

PS-II includes all of the blocks in Fig. 3. The main difference between the PS-I and the PS-II is clearly observed at the maximum likelihood detection stage. Distinct distance calculators are needed for each of the two wires for the MRC. Besides, while Alamouti coding is used, distance calculations and the decision are made after the linear combiner, and therefore there is no need of using a distance calculator for each of these two wires. The receive vectors of the PS-II are given below.

$$
\begin{gathered}
\mathbf{Y}_{1 M}=\mathbf{H}_{1}^{t}\left[\begin{array}{c}
S_{1 R, 0} \\
S_{1 R, 1} \\
\vdots \\
S_{1 R, N-1}
\end{array}\right]+\mathbf{N}_{1}, \\
\mathbf{Y}_{2 M}=\mathbf{H}_{2}^{t}\left[\begin{array}{c}
S_{1 R, 0} \\
S_{1 R, 1} \\
\vdots \\
S_{1 R, N-1}
\end{array}\right]+\mathbf{N}_{2}, \\
\mathbf{Y}_{3 M}=\mathbf{H}_{3}^{t+m T}\left[\begin{array}{c}
S_{1 I, 0} \\
S_{1 I, 1} \\
\vdots \\
S_{1 I, N-1}
\end{array}\right]+\mathbf{N}_{3}, \\
\mathbf{Y}_{4 M}=\mathbf{H}_{4}^{t+m T}\left[\begin{array}{c}
S_{1 I, 0} \\
S_{1 I, 1} \\
\vdots \\
S_{1 I, N-1}
\end{array}\right]+\mathbf{N}_{4} .
\end{gathered}
$$

$\mathbf{Y}_{1 M}$ and $\mathbf{Y}_{2 M}$ are received simultaneously by wires 1 and 2 at time $t$, respectively. $\mathbf{Y}_{3 M}$ and $\mathbf{Y}_{4 M}$ are received simultaneously in a similar way at time $t+m T$. The detection of symbol $S[k]$ is obtained as following:

$\widetilde{S}_{1}[k]=\min _{S_{n}}\left(\left|\mathbf{Y}_{1 M}-\mathbf{H}_{1}^{t}\left[\begin{array}{c}\operatorname{Re}\left\{S_{n}\right\} \\ 0 \\ \vdots \\ 0\end{array}\right] \|^{2}+\mathbf{Y}_{2 M}-\mathbf{H}_{2}^{t}\left[\begin{array}{c}\operatorname{Re}\left\{S_{n}\right\} \\ 0 \\ \vdots \\ 0\end{array}\right]\right|^{2}+\right.$

$\left.\left|\mathbf{Y}_{3 M}-\mathbf{H}_{3}^{t+m T}\left[\begin{array}{c}j \cdot \operatorname{Im}\left\{S_{n}\right\} \\ 0 \\ \vdots \\ 0\end{array}\right]\right|^{2}+\left|\mathbf{Y}_{4 M}-\mathbf{H}_{4}^{t+m T}\left[\begin{array}{c}j \cdot \operatorname{Im}\left\{S_{n}\right\} \\ 0 \\ \vdots \\ 0\end{array}\right]\right|^{2}\right)$

The proposed systems do not have any data rate loss due to the fact that they get diversity gain. If any diversity method were not applied, $N$ symbols would be transmitted in $T$ seconds by $N$ subcarriers. The system-I provides

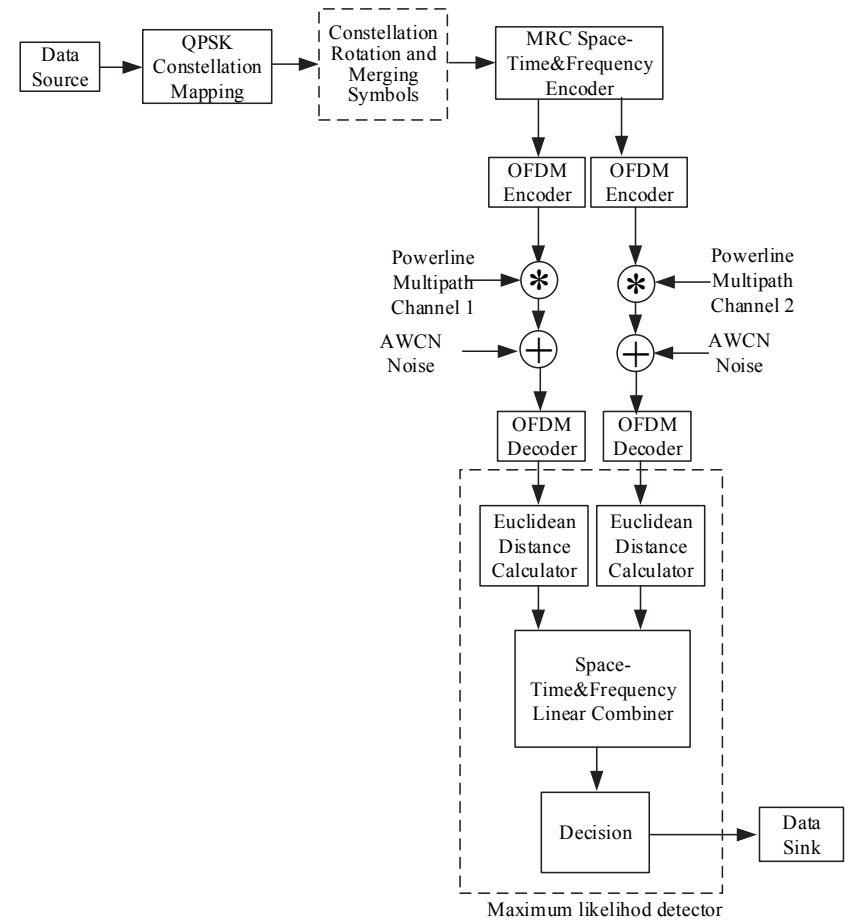

Fig. 3. Block diagram of space-frequency or space-timefrequency diversity systems as Maximal Ratio Combining in use.

transmitting $4 N$ symbols in $4 T$ seconds and the proposed system-II provides transmitting $2 N$ symbols in $2 T$ seconds, hence there is no data rate loss. Conventional systems do not include constellation rotation and symbol merging block in Fig. 3. MRC Space-Time \& Frequency Encoder in Fig. 3 encodes symbols to obtain; space-frequency, rate $1 / 2$ space-time-frequency or the proposed rate-1 space-timefrequency diversity.

\section{Simulation Results}

In the simulations, $\sigma^{2}$ is normalized to $1 /(2 S N R)$ per complex dimensions and the rejection method is used to map a uniform random variable to Middleton Class A random variable [20]. The $\Gamma$ and $A$ values for Middleton Class A random variable are equal to 0.1 and used $m$ values are $0,1,2, \ldots, 9$. The OFDM parameters are $f_{c}=30 \mathrm{MHz}$, $\Delta f=1 \mathrm{kHz}, N=1024$. The channel is assumed as being known at the receiver. 4-QAM and 16-QAM constellations are used for rate 1 and rate $1 / 2$ diversity methods respectively to keep transmission rate equal to each other.

Figure 4 shows the SNR-BER performances of rate 1 diversity methods that applied to power line communication. Because of the proposed systems yielding both frequency and time diversity, they have higher order diversity level than space-frequency diversity applied systems. Hence, the BER value decreases exponentially with diversity order and SNR; the gain advantage of the proposed systems can be expected to increase more by SNR level. Since constellation rotation causes to carry information by amplitudes in two complex dimensions, it has an error 
disadvantage compared to non-rotated QPSK constellation. Therefore the second order diversity (space-frequency Alamouti and space-frequency maximal ratio combining diversity) applied systems have a gain advantage over the proposed systems as SNR level under $12.5 \mathrm{~dB}$. However owing to higher order diversity level, the proposed systems have an increasing gain advantage as the SNR level above $12.5 \mathrm{~dB}$.

Figure 5 shows the SNR-BER performances of fourth order diversity methods for power line communication. The proposed systems have a transmission rate advantage over the other fourth order diversity systems. In order to make transmission rate equal to each other 4-QAM and 16QAM constellations are used for the rate 1 STF and rate $1 / 2$ STF diversity systems respectively. The proposed systems can have more than $6 \mathrm{~dB}$ gain over the other STF diversity systems (rate $1 / 2$ STF Alamouti and rate $1 / 2$ STF maximal ratio combining).

\section{Conclusions}

Applying fourth order full rate space-time-frequency diversity methods to indoor power line communication is showed in this paper. Yielding STF coding as transmit and receive diversity for PLC are presented. The proposed systems are simulated for PLC channel and impulsive noise conditions. The proposed systems are compared with other space-frequency and STF coding is applied power line communication systems under same conditions. According to simulation results, the proposed systems need a threshold SNR level to have a gain advantage over SF system. However, the BER value decreases exponentially with diversity order at high SNR levels. Therefore, owing to the higher order of diversity level, the gain advantage of the proposed systems is expected to increase more against $\mathrm{SF}$ systems by SNR. The proposed systems keep data rate full without increase in detection complexity. The performances of proposed full rate STF coded; receive diversity system (PS-II) and transmit diversity system (PS-I) are similar to each other. However, the cost of transmitting and receiving units may differ from each other. The other STF diversity applied PLC systems in the literature reduce data rate to half and therefore the proposed systems can be referred to as rate 1 and the other STF systems can be referred to as rate $1 / 2$. The proposed STF systems can have $6 \mathrm{~dB}$ more gain than the rate $1 / 2 \mathrm{STF}$ techniques by using transmission rate advantage. On the other hand if the transmission rates are reduced to half by transmitting twice the transmit symbols, the diversity order can be increased twofold with the proposed systems.

\section{References}

[1] ZIMMERMANN, M., DOSTERT, K. A., et al. Multipath model for the powerline channel. IEEE Transactions on Communications, 2002, vol. 50, no. 4, p. 553-559. DOI: $10.1109 / 26.996069$
[2] WEINSTEIN, S. B., EBERT, P. M., et al. Data transmission by frequency-division multiplexing using the discrete Fourier transform. IEEE Transactions on Communication Technology, 1971, vol. 19, no. 5, p. 628-634. DOI: 10.1109/TCOM.1971.1090705

[3] ZIMMERMANN, M., DOSTERT, K. A. Analysis and modeling of impulsive noise in broad-band powerline communications. IEEE Transactions on Electromagnetic Compatibility, 2002, vol. 44, no. 1, p. 249-258. DOI: 10.1109/15.990732

[4] PROAKIS, J. G., SALEHI, M. Digital Communications. $5^{\text {th }}$ ed. New York: McGraw-Hill, 2007. ISBN: 9780072957167

[5] GIOVANELI, C. L., YAZDANI, J., FARRELL, P., HONARY, B. Application of space-time diversity/coding for power line channels. In Proceedings International Symposium on Power Line Communications Applications. Athens (Greece), 2002, p. 101-105

[6] VERONESI, D., RIVA, R., BISAGLIA, P., et al. Characterization of in-home MIMO power line channels. In IEEE International Symposium on Power Line Communication and Its Application. Udine (Italy), 2011, p. 42-47. DOI: 10.1109/ISPLC.2011.5764435

[7] GIOVANEl, C. L., HONARY, H., FARRELl, P. Spacefrequency coded OFDM system for multi-wire power line communication. In Proceedings of IEEE Power Line Communication and Its Application ISPLC 2005. Vancouver (Canada), 2005, p. 191-195. DOI: 10.1109/ISPLC.2005.1430494

[8] ADEBISI, B., ALI, S., HONARY, B. Space-frequency and spacetime-frequency M3FSK for indoor multiwire communications. IEEE Transactions on Power Delivery, 2009, vol. 24, no. 4, p. 2361-2367. DOI: 10.1109/TPWRD.2009.2028495

[9] TAROKH, V., JAFARKHANI, H., CALDERBANK, A. R. Space time block codes from orthogonal designs. IEEE Transaction on Information Theory, 1999, vol. 45, no. 5, p. 1456-1467. DOI: 10.1109/18.771146

[10] JAFARKHANI, H. A quasi-orthogonal space-time block code. IEEE Transaction on Communications, 2001, vol. 49, no. 1, p. 1 to 4. DOI: $10.1109 / 26.898239$

[11] ALAMOUTI, S. A simple transmit diversity technique for wireless communications. IEEE Journal on Selected Areas in Communications, 1998 , vol. 16, no. 8, p. 1451-1458. DOI: 10.1109/49.730453

[12] HACIOGLU, G., GANGAL, A. A rate-1 space-time diversity method for rotated 4-qam constellations. Wireless Personal Communications, 2012, p. 211-223. DOI: 10.1007/s11277-010-0180-8

[13] HACIOGLU, G., GANGAL, A. A transmit diversity method for DVB-H and IEEE 802.20. IEEE Communications Letters, 2011, vol. 15, no. 12, p. 1356-1358. DOI: 10.1109/LCOMM.2011. 101911.111768

[14] CHOE, S., YOO, J. Space-time/space-time-frequency-coding based MIMO-OFDM over power line channels. IEEE Electronics Letters, 2012, vol. 48, no. 16, p. 999-1000. DOI: 10.1049/el.2012.1786

[15] MENG, H., GUAN, Y. L., LAW, C. L., et al. Modeling of transfer characteristics for the broadband power line communication channel. IEEE Transactions on Power Delivery, 2004, vol. 19, no. 3, p. 1057-1064. DOI: 10.1109/TPWRD.2004.824430

[16] GALLI, S., BANWELL, T. C. A deterministic frequency-domain model for the indoor power line transfer function. IEEE Journal on Selected Areas in Communications, 2006, vol. 24, no. 7, p. 1304 to 1316. DOI: $10.1109 /$ JSAC.2006.874428

[17] TONELlO, A. M., VERSOlATtO, F., BÉJAR, B., ZAZO, S. A fitting algorithm for random modelling the PLC channel. IEEE Transactions on Power Delivery, 2012, vol. 27, no. 3, p. 1477 to 1484. DOI: 10.1109 /TPWRD.2012.2196714

[18] TLICH, M., ZEDDAM, A., MOULIN, F., GAUTHIER, F. Indoor power-line communications channel characterization up to $100 \mathrm{MHz}$. Part I: One-parameter deterministic model. IEEE Transactions on Power Delivery, 2008, vol. 23, no. 3, p. 1392-1401. DOI: 10.1109/TPWRD.2008.919397

[19] MIDDLETON, D. Statistical-physical model of electromagnetic 
interference. IEEE Transactions on Electromagnetic Compatibility. 1977, vol. 19, no. 3, p. 106-126. DOI: 10.1109/TEMC.1977. 303527

[20] TRANTER, W. H., SHANMUGAN, K. S., RAPPAPORT, T. S., KOSBAR, K. L. Principles of Communication Systems Simulation with Wireless Applications. $1^{\text {st }}$ ed. New Jersey: Prentice Hall, 2004. ISBN: 9780134947907

\section{About the Author ...}

Gokce HACIOGLU received B.Sc. degree in Electronics
Engineering from Karadeniz Technical University, Trabzon, Turkey, in 2000, the M.Sc. and Ph.D. degree in Electrical and Electronics Engineering with majors in wireless communication diversity techniques from Karadeniz Technical University Graduate School of Natural and Applied Sciences, Trabzon, Turkey in 2005 and 2011, respectively. His current research interests include diversity methods, routing algorithms of wireless sensor networks (WSN), intelligent transport systems, visible light and power line communications and their applications in sensor networks.

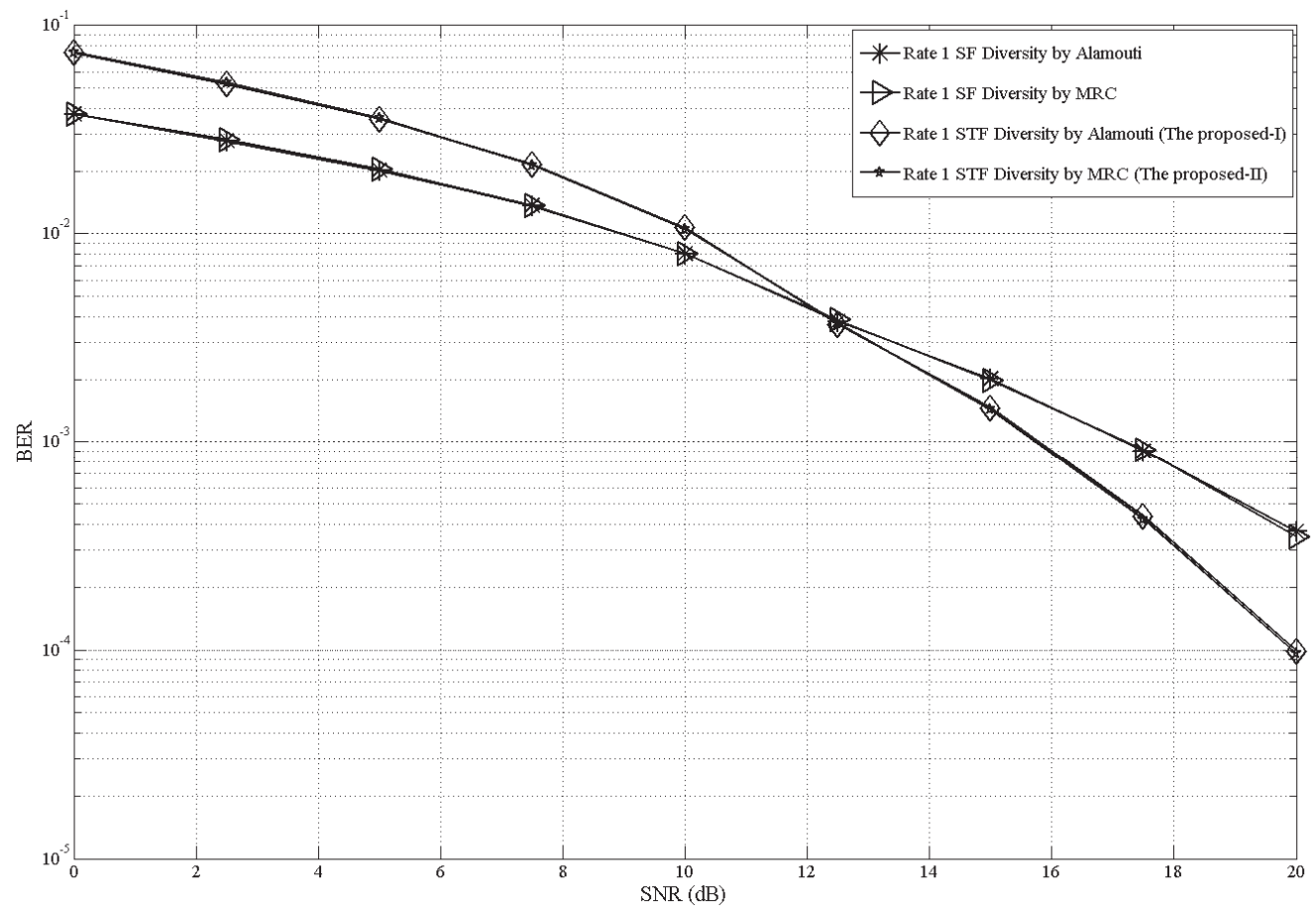

Fig. 4. SNR-BER performances rate 1 space-frequency diversity applied and the proposed systems.

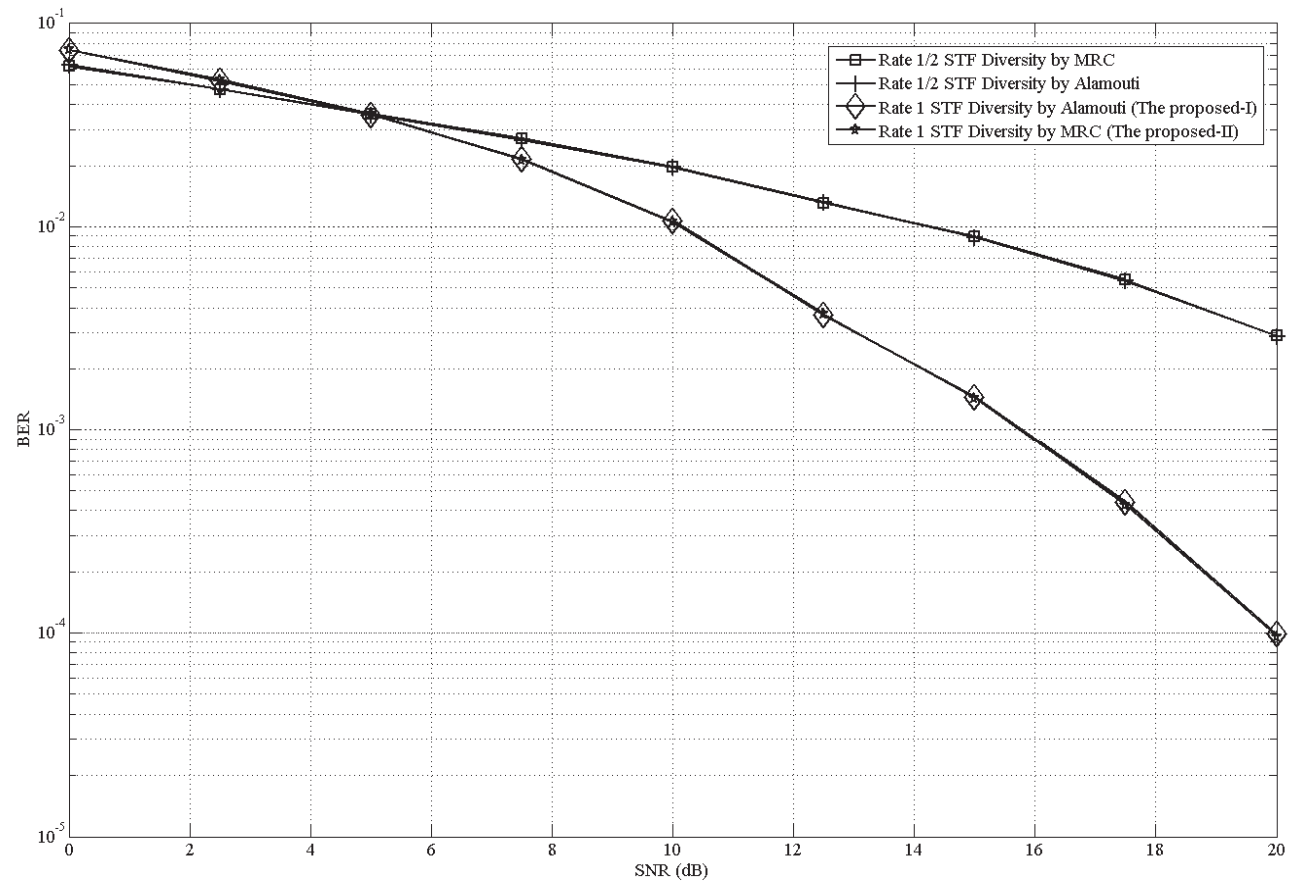

Fig. 5. SNR-BER performances of Space Time Frequency Diversity applied power line communication systems. 